\title{
Comments on: Transversality of the Shapley value
}

\section{Stef Tijs}

It is a good tradition of TOP to invite experts in OR and game theory to write a survey on a special topic. These papers are also interesting for readers who are not specialists in the treated field but may have influence on their further research. For cooperative game theory, in the past, there were survey papers on the compromise value (Tijs and Otten 1993) and on Operations Research Games (Borm et al. 2001), and these papers were frequently cited and inspired to new research. Now here is the paper by Moretti and Patrone on the Shapley value. As we can see in their list of references, in the fifty five years of the existence of the Shapley value, there have been not only many follow ups but also many surveys. This paper also gives a good survey of the many theoretical contributions in the past. But the feature of this survey is the eye for applications. Many applications are well known just as the Shapley-Shubik index in voting situations and the Baker-Thompson rule in landing fee problems, where users of a piece of the strip contribute equally to the costs. However, contributions of the Shapley value in reliability theory and genetics are much less known. They are treated in this paper extensively, and they may inspire scientists in different other disciplines also to apply the Shapley value in new interactive situations. I think the authors did an excellent job in writing a beautiful survey of topics around the Shapley value and I expect that, inspired by this work, new practical situations will be found where Shapleys ideas can again be useful.

This comment refers to the invited paper available at: http://dx.doi.org/10.1007/s11750-008-0044-5.

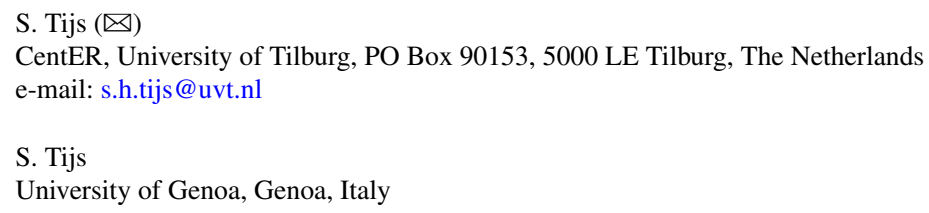




\section{References}

Borm PEM, Hamers HJM, Hendrickx RLP (2001) Operations research games: a survey. Top 9:139-199

Tijs S, Otten GJ (1993) Compromise values in cooperative game theory. Top 1:1-51 